\title{
Algunos Datos del Comercio \\ Precolombino en Colombia
}

Por

S. HENRY WASSEN 


\section{PREFACIO}

Durante mi estadía en Colombia en 1955 para unas investigaciones etnográficas, el señor Director del Instituto Colombiano de Antropología, doctor Antonio Andrade Crispino, ha tenido la gran amabalidad de invitarme a colaborar en la Revista del Instituto que tan dignamente dirige. Como tema de mi humilde contribución he escogido el comercio precolombino, principalmente en la región del continente suramericano que ahora ocupan Colombia y algunas otras repúblicas vecinas.

Mis primeros estudios para este trabajo datan más o menos desde el año de 1930, cuando tuve el placer de escuchar las conferencias acerca del comercio entre los indios suramericanos, dictadas por mi maestro, el barón Erland Nordenskiold, catedrático de la Universidad de Gotemburgo. Mi compilación de datos de la literatura americanista fue continuada después de la muerte de Nordenskiold en el año de 1932, pero nunca logré llevar a cabo un trabajo que ya había intentado. La terminación de otras obras, mi primer viaje a Colombia de 1934 a 1935 y la guerra mundial me obligaron a abandonar por completo el material manuscrito que tenía en proyecto. Ahora, ya, en la misma tierra colombiana, en proporción de mis posibilidades durante una corta estadía en Buenaventura, con sólo algunas anotaciones en mi poder y sin las ricas posibilidades de la biblioteca americanista del Museo Etnográfico de Gotemburgo, me ha sido en todo casi una tarea muy difícil pero honrosa para cumplir con este artículo, según los deseos y la invitación del doctor Antonio Andrade Crispino, a quien le agradezco muy de veras la aceptación de mi modesta colaboración en la Revista del Instituto Colombiano de Antropología como contribución de mi parte a tan valiosa Revista.

Aprovecho esta ocasión para dar las gracias a mi distinguido amigo el doctor Luis Torrijos Blanco, por su importante aporte en la redacción de este artículo.

Buenaventura, marzo de 1955.

El autor 


\section{Consideraciones generales}

En un artículo llamado Indianischer Handel el americanista Th. Koch-Grünberg trató en 1916 sobre el comercio de algunas tribus suramericanas en el Brasil y en la Guayana, debido a las posibilidades que tuvo de conocer durante sus viajes ese continente. Relata primero su encuentro en 1911 con un grupo de los entonces todavía temerosos indios sirianá. Encontró a los indios en el salto Purumamé y logró iniciar un comercio por trueques con ellos. Después de relatar esto, el autor da su opinión acerca del indio como comerciante, en las palabras siguientes: "Der Indianer ist ein geborener Handelsmann. Auch im primitivsten $\mathrm{Zu}$ stañde, fern von europäischem Einfluss, nimmt er jede Gelegenheit wahr, um Tauschhandel zu treiben, und treibt ihn mit Ausdauer, ja mit Leidenschaft".

En su trabajo (Koch-Grünberg, 1916), el autor después da unos ejemplos de sus experiencias en el estudio del comercio indígena, como la especialización en ciertos artículos y la importancia de los largos y muchas veces muy peligrosos caminos de comercio. Como un ejemplo, dice que el curare fuerte de los piaroa en el medio curso del Orinoco, a veces llegó a tribus que vivían más de 1.000 kilómetros de la tierra de origen. Nos relata muchos productos de los indios en el Brasil como los "ralladores" para la preparación de yuca, hechos por los indios yekuaná en el alto Orinoco, de los cuales tenían gran cantidad y los vendieron. Estos indios viajaron de un pueblo a otro cargados con estos "ralladores" y recibieron en cambio, especialmente perros de caza. Estos perros llegaron de los arekuna, los cuales los recibieron de los ackawoi, que habitaban más al Este y tenían relaciones con los ingleses. Los yekuaná tanto como los makú entre Uraricuéra y Orinoco son caracterizados por Koch-Grünberg como los comerciantes más activos en toda la región: "Alljährlich, Ende Janvar, in den hoechsten Trockenzeit, wenn der Tiefstand der Flüsse die Schiffahrt erlaubt, besuchen sie in leichtem Kahn die 
befreundeten Stämme im fernen Osten auf den Savannen Guayanas. Sie müssen dabei durch das Land der Schirianá und Waika, ihrer Todfeinde, fahren und sind nie vor ihren Ueberfällen sicher. Wochen-, ja monatelang bleiben sie der Heimat fern, und wenn sie überhaupt zurückkehren, so sind ein paar europäische $W$ aren von geringem $W$ ert der Lohn für die vielen Anstrengungen und Gefahren der weiten Reise". (Koch Grünberg, 1916, p. 159). Lo que dice Koch-Grünberg aquí, si nos vamos a la literatura del tiempo de los descubrimientos, pudiéramos compararlo con lo que dice Oviedo (1853, t. III, p. 140) cuando habla de los indios de la provincia de los cueva:

"Quando los indios no tienen guerra, todo su exercicio es tractar é trocar quanto tienen unos con otros; é assi de unas partes á otras los que viven en las costas de la mar o por los ríos, van en canoas a vender de lo que tienen complimento é abundancia, é ácomprar de lo que les falta. É assimesmo tractan por la tierra, é llevan sus cargas a cuestas de sus esclavos: unos llevan sal, otros mahiz, otros mantas, otros hamacas, otros algodón hilado ó por hilar, otros pescados salados; otros llevan oro (al qual en la lengua de Cueva llaman yrabra). En fin, aquello que les falta á los indios es lo que más estiman, é aun algunos venden los proprios hijos. É todas estas cosas é otras se dan unas á trueco de otras, porque no tienen moneda ni cierto prescio, é assi acaescen en esta manera de cambiar muchos engaños, é que se dan cosas que valen poco, por las que valen mucho más."

\section{Artículos con que se comerciaba en Sur América}

Cuando Erland Nordenskiold en Gotemburgo dictó sus conferencias acerca del comercio de los indios, lo hizo como preparación de un trabajo científico. Debido a su muerte en 1932, el trabajo fue suspendido, según yo sé, antes de principiar a escribirlo. Recuerdo muy bien cómo trabajaba con un mapa en que quería marcar artículos de comercio en las regiones de las cuales se hizo mención en la literatura. Su interés principal se relacionó con las materias primas y con el comercio de ellas. Tenemos, por ejemplo, el comercio de lana de llama en el Perú, comercio este comprendido de la altiplanicie a la costa. Otros productos comerciales en el Perú eran los remedios. Los curanderos de la región 
del Cuzco se fueron por largas distancias con sus remedios y medicinas. El curare era en regiones considerables del Norte de Sur América una mercancía de gran importancia como también el urucú y la cera. Comerciaban además con plumas, así por ejemplo, los mojo recibieron plumas de los yuracáre. Los metales tenían una importancia considerable: el oro en el Oeste y Noroeste del continente, en las regiones al Este del Cuzco y al Noreste de Cochabamba. El cobre se hallaba también en el Oeste. Había cobre puro en la región de Tiahuanaco. El estaño ofrecía también mucho interés. Pero llegó todo el estaño de la región del lago Titicaca? se preguntó Erland Nordenskiold. El comercio de bronce, sin duda muy importante, pasó por la costa peruana al Ecuador, pero parece que no llegó a Colombia. De su centro incaico el bronce llegó también a Chile y a la Argentina. Como acabo de decirlo, Nordenskiold se interesaba mucho por el estaño. No existen, nos dijo, pruebas para que los indios en México nunca entendieron fundir el mineral y según la opinión de Rivet los objetos de bronce hallados en México debieron ser traídos de América del Sur. Las piedras preciosas eran también un artículo con que comerciaban los indios en tiempos precolombinos. Las piedras verdes les gustaron mucho y las turquesas eran un producto comercial. Nordenskiold consideraba a Puna de Atacama como una región posible para los orígenes de turquesas.

A los productos finos con que se comerciaba a largas distancias se agregan también las piedras nefríticas, o jade, piedras que uno no puede distinguir macroscópicamente una de otra. $\mathrm{Pa}$ rece que se localiza el origen de estos minerales en algunos lugares del Este del Brasil. Y probablemente los indios tapajó, de los cuales trata Heriarte en su obra sumamente rara (1874), recibieron el material de esas regiones brasileñas. La piedra nefrítica de color verde oscuro se halla en las Antillas y puede ser distinguida de la de color verde más claro, la que es conocida en las Antillas del Sur y que tiene que ser importada allá. Según Oviedo los indios arawak viajaron de la costa del Norte por los ríos, tierra adentro, a buscar las piedras verdes.

Eran muchos los productos que señalaba Nordenskiold en su mapa. Yo hice notar algunos, por ejemplo, en Colombia el pescado, el oro, la ropa, la sal, las esmeraldas y las cuentas de caracol; en el Brasil el curare, las bodoqueras, los ralladores de yuca, los perros, las canoas, los canaletes y las hachas de piedra; 
en Venezuela y la Guayana las canoas, los adornos de plumas, y en fin, para trasladarnos al Sur hasta la Tierra del Fuego, manteca de ballena, pirita, piel de zorro, carne y piel de guanaco así como pieles y manteca de lobo. Es de lamentar muy profundamente que el catedrático Erland Nordenskiold, que tenía un verdadero conocimiento de todo lo relacionado con los indios suramericanos, no hubiera tenido tiempo para comenzar el trabajo de que hago aquí otra vez mención. Sin embargo, ya en su obra en sueco de 1912, con relación a la historia cultural de los indios suramericanos, Nordenskiold dedicó el capítulo 20 al comercio de los indios. Aprovecho la oportunidad para incluir en esta obra unas partes de una traducción al inglés del trabajo de Nordenskiold. La traducción fue hecha en Berkeley en 1926 cuando Nordenskiold estaba en la Universidad de California dictando conferencias:

"It is a great mistake to believe that the Indian is always on the war-paht. There are, it is true, Indian tribes living in a constant state of war with each other, in a struggle for life and death, so that no peaceful communication is possible. However, peace rather than war is the normal condition between tribes, even though it be armed peace as between "civilized" states. A lively trade is carried on between them. Still, it is not necessary for two tribes to be peaceably inclined toward each other for them to trade together. Thus, Im Thurn relates that in Guiana, Indian traders can pass unmolested through a hostile district. When we speak of a hostile district, we must remember that the Indian states, even if they only count a few hundred inhabitants, have their defined geographical boundaries." .... "Among the Indian tribes on the Rio Xingú, Karl v.d. Steinen found a very brisk trade going on. Every tribe had a speciality in which it dealt. Trading with intermediaries also existed here. According to v.d. Steinen, they did not understand barter on the Rio Xingú (i.e. object for object), but the trade there was an exchange of friendship's gifts, though on the principle that there should be no meanness on either side."

En la continuación mucho se ha alterado si uno compara el libro sueco con la traducción inglesa, sin duda por la razón de que el mismo Nordenskiold modificó su texto. Lo que sigue, traducido de las páginas 124 y 125 de la edición sueca, lo considero como cosa importante para cualquier estudio del comercio de los 
indios precolombinos: "Trade has, of course, done much to spread cultural influences among tribes. It is, therefore, of the greatest interest to study the trade routes and radii of different tribes. It grows increasingly difficult to get an opportunity of oserving Indian trade in its original form, for the white man pushes his way in everywhere and isolates the tribes which are still independent or at least preserve most of their old culture, from one another. But there was also a considerable limitation of trade even in older times. There was probably never any direct commercial intercourse between the Quichua in Perú and the Chibcha in Colombia. Nor did any trade routes penetrate the great forests to the east of Cuzco, the capital of the Inca Empire."

Como es bien sabido, la sal era un producto muy importante en el intercambio indio, tanto en Colombia como en otras partes del continente. Sin embargo, uno de mis colegas en Suecia ha comenzado un estudio de la distribución y comercio de la sal en América del Sur y por eso no trataré dicho comercio muy en detalle en este trabajo. En parte se trataba de sal sólida como en Colombia, donde forma "bancos en Zipaquirá, Sesquilé, Nemocón, Upía y el Valle de Tenza, en la cordillera oriental," (Estiliano Acosta, 1941), en parte de sal en disolución "dando origen a fuentes salinas que provienen de los lugares en donde se hallan los bancos o las margas salíferas; estas fuentes son frías. En la cordillera central (Ríogrande, Supía, Guaca, etc.) se encuentran fuentes termales que tienen su origen en rocas profundas (rocas eruptivas), y son ricas en sal. Las salinas marítimas son muy numerosas." (Estiliano Acosta, 1941). En Colombia, las minas de sal en Zipaquirá, han sido explotadas desde tiempos prehispánicos. Salina Grande en Puna de Jujuy, en Argentina, es un ejemplo de un depósito muy grande de sal de laguna. También en Tiahuanaco había mucha sal.

En septiembre de 1941, mi compatriota, el señor George Dahl, ahora establecido en Sincelejo, tuvo la bondad de informarme acerca de unas fuentes de sal conocidas personalmente por él. Quiero publicar los nombres aquí pensando que los datos tal vez sean de interés en comparación de las informaciones dadas por los cronistas. Según dicho señor, hay fuentes saladas en un lugar llamado El Chupadero en Antioquia, entre Santa Rosa de Osos y Hoyo Rico. (Las palomas de la región montañosa se van por allá a beber). 
En el Este de Antioquia hay una fuente en el río San Juan Bartolomé donde desemboca el río Volcán. Otra fuente se encuentra más abajo en Puerto Regla. Una fuente con mucha sal y muy fuerte hay en el río La Miel, en Caldas, más o menos a una legua río arriba de donde desemboca el río Manso en el río La Miel.

En el Chocó me informaron que Tadó en el río San Juan significa "río de sal" y parece que pasó por aquí una de las vías comerciales de distribución de sal en esa región.

Cronistas como Oviedo y Cieza de León mencionan varias veces el comercio de sal, la localización de las fuentes, etc., cosa que es bien sabida. Por ejemplo, una información típica de Oviedo es lo que dice en el tomo II de su Historia General (p. 334) respecto de que Isla Fuerte, "en frente de Caporoto é del río del Cenú" era una isla llana "donde se hace mucha sal". Cieza menciona fuentes de sal tanto en Caramanta como en Cartago (1862, cap. XXXV). Su información dice que Ancerma tenía su nombre de la palabra ancer por sal (1862, cap. XVI, p. 368), la que ha sido repetida varias veces. Oviedo tiene la misma información (1855, p. 142).

En la literatura se hace mención también de ceniza salada como producto comercial (en Amazonas). La literatura sobre datos de interés relativos al comercio indio es bastante rica. Además de los trabajos indicados en la primera sección de la bibliografía, he tratado de referirme en la segunda sección de la misma a otras obras que contienen datos importantes acerca del comercio indígena. Algunos de los trabajos citados ya son especializados como los de Latcham (1909) y los de Koch-Grünberg (1916). Aquí puede ser mencionado un trabajo popular en sueco (Lindblom, 1931), por tener también algunos datos de América.

Hago aquí mención de algunos trabajos de interés, para un entendimiento de todo lo que se relaciona con el trato y el comercio en los pueblos indígenas, tanto en América como en otras partes del mundo. Lasch (1906) ha tratado el sistema de ferias y también da ejemplos de México antiguo (pp. 714, 766-768, 772 y 778). Fr. Graebner (1910) trata también del comercio en México (p. 186 y sig.). En el mismo trabajo se ocupa también del trato de los indios en el Brasil, en la Guayana, etc. En el capítulo XXXII de la obra de Walter E. Roth (1924) se halla un enorme material con muchos datos detallados. Hoyt (1926) en un trabajo moderno y bueno nos da información de muchos 
problemas relacionados con el comercio primitivo, por ejemplo, el origen de los conceptos de valor (p. 5), el concepto de propiedad (p. 71), señales de propiedad (p. 81), trueque (pp. 82-83), moneda (p. 85), regalos (p. 97 y sig.), el llamado comercio silencioso (p. 111). Dicho autor opina que el comercio en el Perú antiguo era bastante limitado (p. 146), lo que me ha sorprendido un poco al leerlo: "Although a civilization such as that of the Incas can grow up with no trade, or very little trade, special conditions are necessary for that. The Incas were well isolated, and the nearest tribes were some of the least developed in the world." Sin duda alguna, tiene razón absoluta el mismo autor cuando opina que el comercio siempre ha sido conectado con alguna difusión considerable de civilización (Hoyt, 1926, p. 146).

\section{Colombia}

También para Colombia existe un trabajo de suma importancia para todos los que quieran tratar sobre el comercio precolombino en el país. Es la obra de Trimborn (1942) con su análisis detallado, basada en los datos suministrados por los cronistas y por otras fuentes históricas. Aun cuando la obra de Trimborn es, sin duda, ya conocida entre los etnólogos colombianos, me permito resumir aquí algo de su contenido.

Según Trimborn, la región noroeste de Colombia, sobre todo Antioquia, ha sido un centro sumamente importante por el comercio precolombino. Los metales y la sal juegan el principal papel y la ropa de algodón no tiene tanta importancia en comparación con dichos productos. Se comerciaba también con esclavos y víveres. Según Trimborn, los testigos oculares nunca mencionan entre los productos comerciales la cerámica, pero no hay duda de que los indios trataban también con ollas, etc. Al enumerar varios productos, los cronistas a veces no son muy detallados y se limitan a mencionar unos pocos terminando con frases como "ó otras cosas" (Oviedo, Sumario, etc., 1877, pp. 508509), "porque naturalmente todos los indios generalmente, más que todas las gentes del mundo, son inclinados á tratar y á trocar y baratar unas cosas con otras; y así, de unas partes á otras van en canoas y de donde hay sal la llevan a donde carecen de ella, y les dan oro ó mantas, ó algodón hilado, ó esclavos ó pescado, ó otras cosas;..." 

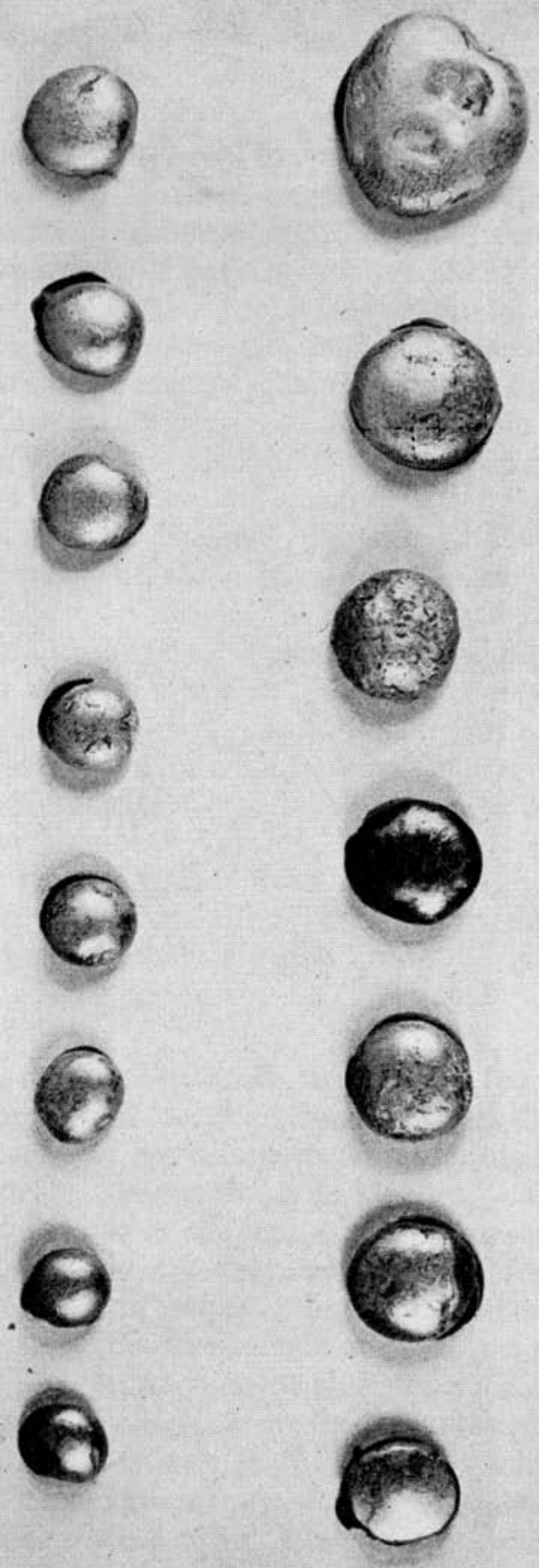

Quince tejuelos colombianos de dos colecciones en Berlín. 
En una fuente considerada como muy importante, la "Descripción de la Ciudad de Tunja" (1610), a donde llegaron los españoles en 1539, se menciona también entre los productos del mercado a la "lova de la tierra", lo que puede ser vasijas de barro.

Trimborn considera a Buriticá, región minera de Antioquia, como un centro de donde salieron cuatro caminos comerciales en varias direcciones. Uno de éstos (op. cit., p. 112) pasó por la cordillera occidental a Urabá hacia las provincias de Nore y Guaca. Este camino debe haber sido muy importante por la comunicación que estableció entre Antioquia y la región del Sur en Centro América. Otro camino (op. cit., p. 114) había de Buriticá al Norte hasta Ituango, pasando por Guacuceco al Sinú, donde tenía conexión con un camino que conduce a las tierras de los tairona al Este. Un tercer camino (op. cit., p. 116) pasó por Pueblo-Llano y Murgia, de ahí a la sierra de Aburrá, es decir, en el alto valle de Porce, y de allí hacia la tierra de los chibcha, de donde pasó al Magdalena en dos ramificaciones, una más hacia el Norte, determinada por White en Yolombó, y otra más hacia el Sur con dirección a R. Negro y Nare. Según White, citado por Trimborn, esto tuvo que ser el camino que comunicaba la tierra de los chibcha con América Central. Al fin, hubo otro camino que condujo, según Trimborn, (op. cit., p. 117) de Buriticá por Cenufana hacia el Este de Caldas hasta el país de los quimbaya. "Por este camino pasó en una dirección oro a medio trabajado de Antioquia, y quizás también algodón crudo, en la otra dirección de la tierra de los quimbaya sal, "mantas" y objetos de oro".

La obra de Trimborn, sumamente concentrada, es una contribución principalísima para nuestro entendimiento del comercio precolombino en regiones muy importantes de Colombia. Trata también de otras cosas, como noticias de puentes, indicadores en piedra de caminos, lugares donde había ferias o mercados, el papel que desempeñaban los comerciantes profesionales, y en fin, se refiere asímismo a otra cuestión importante como es la de saber si había en Colombia precolombina algo que pudiera ser definido como moneda con un valor en sí, independiente del valor del producto mismo. Trimborn niega la existencia en Colombia precolombina de moneda en este sentido, esto sin duda con toda razón. El catedrático Trimborn y yo hemos discutido el asunto por correspondencia cuando yo en 1941 me interesaba por las 
informaciones de Vicente Restrepo (1895, p. 126) cuando dice que "fueron los chibcha con los habitantes del Chimú, en el Perú, los dos únicos pueblos del Nuevo Continente que se sirvieron de moneda para sus cambios. Consistía la moneda de los chibcha en unos tejuelos ó discos de oro, vaciados en moldes apropiados, y sin ninguna señal".

Además, tenemos en Castellanos (1852, p. 507) la información de que entre los Catío "si venden un esclavo por chagualas, de cuyo valor tienen certidumbre", etc., Restrepo sin duda ha aceptado, sin crítica, la opinión de Uricoechea (1854, p. 26) de que "la moneda consistía en ciertos tejuelos de oro fundidos en un molde normal, sin marca ni seña alguna".

Chagualas o tejuelos no eran moneda en el propio sentido de la palabra. Eran ciertos ornamentos o pedazos de oro que tenían en sí cierto valor en el intercambio de productos, más o menos como el cacao tenía su valor en México y en América Central. "El cacao ó almendras que corren por moneda en aquellas partes" (Nicaragua, Oviedo, 1855, t. IV, lib. XLII, cap. IV). "Hay mugeres públicas que ganan é se conceden á quien las quiere por dies almendras de cacao de las que se ha dicho ques su moneda" (Oviedo, 1855, t. IV, p. 37). Fuera del cacao, las "cuentas de piedra" eran un medio de pago en México. De ahí que relate Diego de Landa (1864, p. 128) que "el officio en que mas inclinados estavan es mercaderia, llevando sal, ropa y esclavos a tierra de Ulua y Tavasco, trocándolo todo por cacao y cuentas de piedra que era su moneda, y con esta solían comprar esclavos y otras cuentas con razón que eran finas y buenas, las quales por joyas trayan sobre si en las fiestas los señores".

Para obtener más informaciones acerca de los tejuelos, en 1941 me puse en contacto con el Museo Etnográfico de Berlín, donde, según Vicente Restrepo (1895, p. 127) debían estar unos "tejuelos" de Colombia en una colección Randall-Sokolosky. A pesar de la guerra, el señor Director de dicho Museo, doctor W. Krickeberg, conocido americanista, logró hallar dichos objetos, de los que amablemente sacó una fotografía junto con otros semejantes de una antigua colección Holtschick del mismo Museo. La fotografía, reproducida aquí, muestra en total 15 "tejuelos" de las dos colecciones de Berlín. El diámetro del pedazo más grande es de $2,1 \mathrm{~cm}$. y del más pequeño de $0.8 \mathrm{~cm}$. En una carta del 6 de noviembre de 1941, el doctor Krickeberg me informaba 
que en la lista original de la colección Randall-Sokolosky no se menciona la palabra "tejuelo" sino que los pedazos de oro son llamados pesos (weights). Doce de los pedazos vienen de la colección más vieja de Holtschick y en el catálogo del Museo se dice que son "oro de momia de Ibagué". Todos los trozos están, como lo explica Restrepo, "sin ninguna señal". Según mi parecer tenemos que considerar estos trocitos de oro simplemente como material crudo dejado por los orfebres.

Asímismo, sobre el problema de los tejuelos estuve tratando con Trimborn, quien concretó su opinión en las siguientes palabras: "Auf Grund der mir vorliegenden Materialien kann ich wohl sagen, dass es ein "Geld" im engeren Sinne, das ohne eigenen Gebrauchswert seine Geltung der oeffentlichen Gewalt verdankte, weder bei den Muisca noch im Caucatale gegeben hat. Insofern war also aller Handel Naturaltausch von Gebrauchsgütern, wie dies für die Chibcha von Bogotá auch Oviedo (1851/55, Bd. II, S. 407) bezeugt. Natürlich war es bei dem regen Handelsverkehr zur Herausbildung bevorzugter Tauschgüter gekommen, deren Annahme man stets sicher war, und zwischen denen sich feste Wertrelationen herausgebildet hatten. Im Caucatal spielten neben Salz die sogenanten "chaguales" eine solche Rolle. Es drängt sich dabei die Frage auf, ob solche Schmuckstücke ein bestimmtes Normgewicht hatten; wenn nicht, muss es daneben genormte Metallgewichte gegeben haben; Wagen sind für das Caucatal literarisch und archäelogisch belegt (für die Catío, Hevéjico und aus Fredonia in Antioquia)".

De vez en cuando se mencionan en la literatura las ferias (México, Colombia, Perú). Para referirme a un ejemplo colombiano podemos recordar a Tunja (Descripción, etc., p. 429): "Hácese mercado en esta ciudad de cuatro en cuatro días; de manera que si un mercado es miércoles, el otro será domingo y el siguiente jueves". Muy probablemente esto ya era una institución antigua en Tunja.

En 1947, durante mi estadía en Santa Marta, el doctor Gerardo Reichel-Dolmatoff muy amablemente me suministró unos datos de centros comerciales en la antigua Colombia como Tamalameque en el bajo río Magdalena y en Ibagué donde los pijao mediaron el comercio entre el reino de los Chibcha y el Cauca. También una tribu, ahora extinta, los guayapo, debió haber tenido ferias. Hizo también mención de Ríohacha, lugar de comercio entre la Guajira y el Magdalena. 
Las referencias de las ferias en el reino de los Chibcha han sido citadas muchas veces. Uricoechea (1854, pp. 26-27) hace mención de Coyaima en el territorio de los Poincos, "llamados por los españoles Yaporogos, del nombre de sus caciques". Allá llevaban sal, esmeraldas, mantas pintadas, joyas de oro, etc. ..." "Otra feria famosa se celebraba en los términos del cacique Zorocotá, en donde después se fundó el Puente Real sobre el río llamado entonces Sarabita, á que concurrían los Chibcha del Norte, los Agataes, Chipataes i los industriosos Guanes, que se proveían de sal en cambio de oro i de mantas i tejidos de algodón de diversas calidades i colores". Según se dice esta feria se celebró alrededor de una piedra grande que por tener plata fue derrumbada por los españoles.

Otra feria mencionada por Uricoechea (op. cit., p. 27) "había en Turmequé, cada tres días, i en ella se veían fuera de los frutos comunes, gran cantidad de esmeraldas de Somondoco, aunque al tiempo del descubrimiento estaba ya bien agotada la mina".

En la obra de 1871 (p. XXXII-XXXIII) basada sobre el manuscrito del Padre Lugo, acerca de la lengua de los Chibcha, Uricoechea repite estos datos de los mercados, añadiendo que mercado era en la lengua chibcha ipta (iepta). "El de Bogotá se llama muyneca".

En la literatura antigua los datos definidos sobre comercio entre tribus mencionadas por su nombre no son muy comunes, antes bien se usan expresiones generales como "tierra adentro", etc. Sin duda hay varias indicaciones determinadas, de algunas de las cuales hago mención aquí:

1) Juan de Castellanos, 1852, p. 507, Relaciones Comerciales entre Nutabaes y Tahamía.

2) Federmann (ed. Ternaux), 1837, p. 99, Comercio entre Indios Xaguas y Caquetíos (sal).

3) Cieza de León (1862, t: II, p. 384), Comercio entre "El Valle del Patía" y "La Provincia de Chapanchita y con otra a ella comarcanas".

Eugenio Ortega (1912) ha tratado sobre las relaciones comerciales entre los Chibcha y los Panche, "a pesar de la constante enemistad". "Los primeros se proveían de sal en Zipaquirá y Nemocón en cambio de oro en polvo, guacamayas, loros y naturalmente frutas y pescado". El mismo autor continúa: “...y como desde la ocupación de la sabana por los españoles, se habían sus- 
pendido dichas transacciones, la suprema necesidad del artículo de la sal contribuyó a que los belicosos panches se sometieron al imperio de los conquistadores, aunque después de haber defendido vigorosamente su libertad".

Antes de tabular algunos datos acerca del comercio precolombino en Colombia, me gustaría recordar unas palabras que Erland Nordenskiold explicó en una conferencia para sus alumnos. Dijo que al contemplar la exportación moderna de Sur América se nota que al considerar los artículos de más importancia como el café, el trigo, los cueros, los guineos, etc., éstos son de un origen no americano y que la localización de la cultura moderna en el continente depende de las relaciones transoceánicas. Esto, añadió, es algo totalmente diferente de las condiciones en Sur América precolombina, absolutamente aislada. Nordenskiold opinaba entonces que si bien existía un comercio intertribal, su radio nunca era muy grande. Sin embargo, hay excepciones, como las que constan de vez en cuando en los arqueólogos, por ejemplo, en el famoso cenote de Chichen Itzá en Yucatán. Es muy interesante leer lo que dice Lothrop (1944, p. 426 y sig.) acerca de los objetos de comercio encontrados en América Central, llegados de otras partes. Según Alfred Kidder II (1944, p. 456) hay "considerable evidencia por un comercio entre puntos tan distintos como México y Perú en tiempos precolombinos".

A continuación doy aquí una lista, no muy detallada, de unos productos mencionados en la literatura de la conquista sobre Colombia y algunas regiones cercanas. Es probable que un estudio detallado, que tome en consideración todos los datos en la literatura antigua, pueda también suministrarnos informaciones de interés desde otros puntos de vista. Por ejemplo, existían algunas tribus que no tenían comercio alguno? Cómo fue limitado el comercio por factores como materias primas, guerras, dificultades de transporte, etc.? Solamente al recoger los datos completos de todos los productos y de todas las regiones sería posible estudiar las variaciones en el comercio por producto y por región.

$\mathrm{Al}$ hacer una lista de prueba como la que sigue, se pregunta uno siempre si no existían también otros productos en el comercio precolombino. Sin duda que sí. Pero por lo que toca a Colombia, no tengo todavía noticia alguna de comercio con miel, mientras Oviedo (t. III, p. 245) expresamente menciona la "contractación de miel" en Chitemal, durante el viaje que Francisco de Montejo hizo a Campeche. 


\section{LISTA PRELIMINAR DE PRODUCTOS COMERCIALES EN COLOMBIA PRECOLOMBINO}

A. Gente:

\section{Categoria}

1) Esclavos.

2) "Ciertos muchachos para sacrificios. Mojas. Chibcha.

B. VIVERES Y SAL:

1) “Cigarras y langostas y grillos"; pescado. Sinú.

2) Camarones secos. "La costa del río Grande", entre Cartagena y Santa Marta.

3) Pescado.

a. San Sebastián, Culata de Cieza de León, 1862, p. 379. Urabá.

b. Cali. Pescado y manteca del pescado.

\section{Citaciones $y$ Notas}

Castellanos, 1852, región de Antioquia:

"Si venden un esclavo por chagualas,

De cuyo valor tienen certidumbre,

En una venta hacen tres igualas:

Una las manos por la servidumbre,

Otra la carne, questas gentes malas

Tienen en esto pésima costumbre,

Otra por la cabeza, que ya muerta

Por honra grande ponen á la puerta."

V. Acerca del comercio con esclavos, carne humana, etc. Trimborn, 1942, pp. 117-119, donde se citan varios casos. Oviedo, 1852, t. II, lib. XXVI, p. 402. Repetido por Vicente Restrepo, 1895, p. 71 .

"Mercaderes chibchas iban á comprarlos á esa lejana provincia, etc. ..."

Oviedo, 1877, p. 509.

"y en el Cenú... y estos hallaron allí muchos cestos del tamaño...; los cuales estaban llenos de cigarras y langostas y grillos; y decian los indios que allí fueron presos que los tenian para los llevar á otras tierras adentro, apartadas de la costa de la mar, donde no tienen pescado..."

Oviedo, 1852, t. II, p. 442.

Cieza de León, 1862, p. 361.

“...; matan en esta laguna infinidad de pescado muy sabroso, que dan á los caminantes y contratan con ello en las ciudades de Cartago y Cali y otras partes; sin lo mucho que ellos dan y comen, tienen grandes depósitos de mucha cantidad de manteca que del pescado sacan." 
c. Sinú. Cangrejos, etc.

4) Huevos de tortuga, manteca. Provincia de Venezuela.

5) Sal. Chibcha.

San Sebastián, Urabá.

Montañas de Abibe. Agua cocida "en grandes ollas", etc.

Región de Maracaibo.

"Canoas cargadas de sal".

"Mahiz á trueco de sal".

Sal "á manera de pilon de açúcar", etc.

C. Animales:

1) "Puercos", es decir, pecaris. San Sebastián, Urabá.

2) Perros. "En Darien, traidos de la costa de Cartagena, de tierra de caribes." Mudos.
López de Gómara, 1877, p. 200 (de la entrada de Enciso).

"Hallaron por las casas muchas canastas y espuertas de palma llenas de cangrejos, caracoles sin cáscara, cigarras, grillos, langostas de las que destruyen los panes, secas y saladas, para llevar mercaderes la tierra adentro, y traer oro, esclavos y cosas de que carecen."

Luis de Cisneros, 1912, pp. 39-40.

"Los huebos, los sacan, y haciendo grandes pilas, unos los secan al Sol para guardarlos, y otros convierten en manteca, de que hacen gran comercio con las naciones de tierra adentro..."

Oviedo, 1852 , t. II, p. 381,382 , 387, los descubrimientos de Quesada por el Río Grande, etc.

P. 407: "Hay en muy grande abundançia de sal é muy buena, y es grand contractaçión la que corre por aquella tierra en esta sal..."

Cieza de León, 1862, p. 361.

Cieza de León, 1862, p. 386.

Comp. Castellanos, T. I, canto II, y Vicente Restrepo, 1895, p. 124.

Oviedo, 1852, t. II, pp. 292-293.

Oviedo, 1852, t. II, pp. 381-382.

Cieza de León, 1862, p. 361.

..."á vender la tierra dentro muchos puercos de los que se crian en la misma tierra, diferentes de los de España, porque son mas pequeños y tienen el ombligo á las espaldas..." (la descripción de Cieza explicada en la edición de Espasa Calpe, 1922, p. 26, "es la glándula dorsal de los pecarís").

Oviedo, 1877, p. 491. 
D. Metales:

1) Oro. "Por el nasçimiento de Meta de la otra parte de las sierras".

"Oro que avia en Bogotá venia de la provincia de Neyva."

Oro en Antioquia, etc.

Convenio comercial entre el señor de Guatabita y los señores de los reinos cercanos por regulación de un cambio de trabajadores de oro y otros trabajadores en proporción: "un platero de Guatabita contra dos de las otras regiones." Este convenio pacífico y comercial ha sido tratado en sus detalles por Trimborn, p. 155. 1930, pp. 47-48, y ibid. 1934,

Oviedo, 1852, t. II, p. 307.

Oviedo, 1852, t. II, p. 400.

V. Trimborn, 1942, y otros.

Castellanos, 1886, pp. 24-25.

"Los Guatabitas por la mayor parte eran artífices de labrar oro, y entre los otros indios reputados por mas sutiles en aquestos usos, y así por las provincias convecinas, ajenas de las deste señorio, andaban muchos de ellos divertidos ganando de comer por sus oficios, sin acudir á las obligaciones debidas al Señor segun sus leyes. El cual, vista la falta que hacian así de renta, como de personas, mandó con gran rigor que todos ellos se recogiesen á sus naturales, $y$ que el Señor que menester hubiese algun oficial dellos en su tierra, por uno diese dos de sus vasallos que con el Guatabita residiesen en tanto que el aurífice faltaba. Y en aquel tiempo, como los señores y principales indios abundaban de pálidos metales, granjeados ya por contratos, ya por otras vias, y su felicidad eran las joyas, ornamentos de vivos y de muertos, fué fácil de cumplir lo que pedia, $\mathrm{y}$ en breve tiempo tuvo Guatabita más de dos mil gandules extranjeros en recompensa de los oficiales, y aumento de sus rentas y tributos; con que tambien crecia la jactancia, diciendo que los más altos señores ya le reconocian obediencia, pues que de sus vasallos se servian."

Oviedo, 1852, t. II, p. 279.

2) Cobre. "Por moneda", "tie- Oviedo, 1877, p. 508. rra de los pemeos". 
E. Esmeraldas, etc. Santa Marta.

Somondoco.

F. Ropa:

De algodón, por ejemplo, entre los chibcha.

G. Armas:

Flechas. Antioquia.

H. Coca:

Chibcha. "...y ciertas mantas y cosas de indios, en que se vieron plasmas de esmeraldas y corniolas y jaspes y calcidonias y zafires blancos y ámbar de roca; todas estas cosas se hallaron donde he dicho, y se cree que de la tierra adentro les debia venir por trato y comercio que con otras gentes de aquellas partes deben tener..."

Vicente Restrepo, 1895, p. 125.

"Los indios de Somondoco cambiaban las esmeraldas por oro, mantas de algodón y cuentas."

Oviedo, 1852, t. II, p. 360.

"El principal rescate es oro y qüentas que en esta tierra se haçen, y ropa mucha de algodón."

Cieza de León, 1862, p. 370.

"Los indios son de pequeños cuerpos, y tienen algunas flechas traidas de la otra parte de la montaña de los Andes, porque los naturales de aquellas partes las tienen."

Piedrahita, 1688, Cap. III, Fol. 20.

“...Hablan pocas palabras, y dormian menos, porque lo mas de la noche gastaban en mascar Hayo, que es la yerva, que en el Perú llaman Coca,... después las ponen en vna vasija de barro sobre el fuego, y tostadas las guardan, ó para el comercio en que fundan su mayor riqueza, ó para el gasto de casa, y familia." 


\section{BIBLIOGRAFIA}

\section{A-OBRAS CITADAS}

Castellanos, Juan de. 1852-Elegias de Varones Ilustres de Indias. (Biblioteca de Autores Españoles, t. IV, 2a. edición). Madrid.

1886-Historia del Nuevo Reino de Granada, tomo I. Madrid.

Cieza de Leon. 1862-La Crónica del Perú. (En Enrique de Vedia, Historiadores primitivos de Indias, tomo II, pp. 344-458). Madrid.

Descripción de la ciudad de Tunja, sacada de las informaciones hechas por la justicia de aquella ciudad en 30 de Mayo de 1610 años. (Colección de Documentos Inéditos del Archivo de Indias, tomo II, pp. 393-448). Madrid.

Estiliano Acosta, Jose. 1941-Estudios de la Naturaleza. (La Escuela Normal, $2^{a}$ época, números 2-3). Bogotá.

Federmann, Nicolas. 1837-Belle et agréable narration du premier voyage aux Indes de la mer océane. (Ed. Henri Ternaux). París.

Graebner, Fr. 1910-Handel bei Naturvolkern. (En Karl Andree: Geographie des Welthandels. Bd. I, pp. 149-218). Frankfurt a.M.

Heriarte, Mauricio de. 1874 (1662)-Descripcao do Estado do Maranhao, Pará, Corupá e Rio Das Amazonas. Vienna d'Austria.

Hoyt, Elizabeth Ellis. 1926-Primitive Trade. Its Psychology and Economics. London.

Kidder II, Alfred. 1944 -South American Penetrations in Middle America. (En The Maya and their Neighbors, pp. 441-459). New York. London. Koch-Grünberg, Theodor. 1916-Indianischer Handel. (Kosmos, Handweiser für Naturfreunde, H. 5). Berlín.

Landa, Diego DE. 1864-Relations des choses de Yucatan. (Texte espagnol et traduction française... par l'abbé Brasseur de Bourbourg. París.

LASCH, Richard. 1906-Das Marktwesen auf den primitiven Kulturstufen. (Zeitschrift für Socialwissenschaft, IX, pp. 619-627, 700-715, 764782). Berlín.

Latcham, Ricardo E. 1909-El comercio precolombino en Chile $i$ otros paises de America. Santiago de Chile.

Lindblom, K. G. 1931-Handelsmetoder bland primitiva folk. (Handel och samfärdsel, pp. 1-25). Uppsala.

Lopez de Gomara, Francisco. 1877-Historia General de Las Indias. (Historiadores primitivos de Indias por Enrique de Vedia, tomo I, pp. 155-294). Madrid.

Lothrop, Samuel K. 1944 -South America as seen from Middle America. (En The Maya and their Neighbors, pp. 417-440). New York. London.

Luis de Cisneros, Joseph. 1912 (1764)-Descripción exacta de la Provincia Benezuela. (Colección de libros raros y curiosos que tratan de América, segunda serie, t. XXI). Madrid.

NoRdensKiold, ERLAND. 1912-De sydamerikanska indianernas kulturhistoria. Stockholm.

Ortega, Eugenio. 1912-Los Panches. (Boletín de Historia y Antigüedades, VII, pp. 657-669). Bogotá.

Oviedo y Valdes, Gonzalo Fernandez de. 1851-55-Historia General y Natural de las Indias, Islas y Tierra-Firme del Mar Océano. Tomos I-IV. Madrid.

1877-Sumario de la Natural Historia de las Indias. (Historiadores primitivos de Indias por Enrique de Vedia, t. I, pp. 473-515). Madrid. 
Piedrahita, Lucas Fernandez. 1688-Historia General de las conquistas del Nuevo Reyno de Granada. Amberes.

Restrepo, Vicente. 1895-Los Chibchas antes de la conquista española. Bogotá.

Roth, Walter E. 1924-An Introductory Study of the Arts, Crafts, and Customs of the Guiana Indians. (38 th Ann. Report of the Bureau of American Ethnology, 1916-1917). Washington.

Trimborn, Hermann. 1930-Das Recht der Chibcha in Columbien. (Ethnonologica, Bd. IV). Leipzig.

1934-La politica en "El Dorado". (Investigación y Progreso, año VIII: 5, Mayo 1934). Madrid.

1942-Der Handel im Caucatal. (Zeitschrift für Ethnologie, Jahrg. 74, pp. 112-126). Berlín.

UricoecheA, Ezequiel. 1854-Memoria sobre las antigüedades Neo-Granadinas. Berlín.

1871-Gramática, vocabulario, catecismo $i$ confesionario de la Lengua Chibcha, según antiguos manuscritos anónimos $e$ inéditos, aumentados $i$ correjidos. (Coal. Lingüística Americana, tomo I). París.

B-OTRAS OBRAS SELECTAS DE VALOR POR SUS INFORMACIONES DEL COMERCIO ENTRE TRIBUS SUDAMERICANAS. CON COMENTARIOS

Acuña, Cristoval DE. 1859-A New Discovery of the Great River of the Amazons (1639). (En Clements R. Markham: Expeditions into the Valley of the Amazons, 1539, 1540, 1639). London (Hakluyt Society). Hay también otras ediciones. Tiene informaciones acerca del comercio entre los aguas, es decir omaguas; curuziraris (especialistas en cerámica); tribus que vivían a lo largo del río Basururú; los tupinambá (28 leguas de la boca del río Madeira), que comerciaban con sal de otra tribu cercana.

Baudin, Louis. 1928-L'Empire socialiste des Inka. París.

P. 169, "le commerce local" y p. 170 "le commerce étranger".

BRüHL, Gustav. 1875-Die Culturvolker Alt-Amerika's. New York.

Tiene una bibliografía excelente.

Frezier. 1732-Relation du voyage de la Mer du Sud aux côtes du Chily et du Perou fait pendant les années 1712, 1713 \& 1714. París.

Tiene informaciones de los puelchet, etc.

Gallardo, Carlos R. 1910-Los Onas. Buenos Aires.

P. 291, "comercio".

Garcilasso de la Vega. 1609-Commentarios Reales, que tratan del Origen de los Yncas, etc. Lisboa.

Otras ediciones también. En el libro VI, cap. XXXV, el autor cita padre Blas Valera recopilando leyes y ordenanzas dictadas por el inka Pachacutec, tiene informaciones del modo de contar en quipus, los contadores, etc.

Im Thurn, Everard F. 1883-Among the Indians of Guiana. London.

Mucha información acerca del comercio de tribus como warrau, macusi, arecuna, wapisiana, "true caribs", etc.

Koch-Grünberg, Theodor. 1923-Vom Roroima zum Orinoco, tomo III, Stuttgart.

Informaciones del interés comercial en las páginas $12,13,50,51,63$, $65,318,319,348-349$. Las páginas $355-357$ son exclusivamente dedicadas al comercio de los indígenas. 
Krause, Fritz. 1911-In den Wildnissen Brasiliens. Bericht und Ergebnisse der Leipziger Araguaya-Expedition 1908. Leipzig.

Pp. 278-280, "Handel, Geld, Masse".

La Condamine, C. DE. 1921-Viaje a la América Meridional. Madrid (Calpe). Informaciones de las piedras de las amazonas, pp. 89-91.

Linne, S. 1925-The technique of South American ceramics. (En Goteborgs Kungl. Vetenskaps. Och Vitter hetssamhalles Handlingar, ser. 4\%, t. 29:5). Goteborg.

P. 169, un mapa sobre la exportación y la importación de cerámica, p. 162 y sig. el texto.

Martius, Carl Friedrich v. 1867-Zur Ethnographie Amerika's zumal Brasiliens. Leipzig.

P. 89 y sig. mucha información del derecho de los indígenas, los bienes de ellos, etc. En p. 622 relata como los arecuna usaban hilos de algodón como medio de cambio o pago, lo que ya observó Colón en las Antillas.

Melin, Douglas. 1929-I Amazonas urskogar. Uppsala.

P. 134, comercio entre los makú.

Metraux, A. 1928-La civilisation matérielle des tribus tupi-guarani. París. Obra extraordinaria para nuestro conocimiento del conjunto de tribus tupi-guarani.

1942-The Native Tribes of Eastern Bolivia and Western Matto Grosso. (Bureau of American Ethnology, Bull. 134). Washington.

P. 18 y p. 78, informaciones del comercio de los mosetene y los mojo, sacadas entre otras cosas del trabajo del Padre Pedro Marbán: Relación de la Provincia de la Virgen del Pilar de Mojos.

Mujia, Ricardo. s. a.-Bolivia-Paraguay. Exposición de los títulos que consagran el derecho territorial de Bolivia, sobre la zona comprendida entre los ríos Pilcomayo y Paraguay. Anexos, tomo I, Epoca Colonial. La Paz.

Pp. 447-481, contiene la Descripción del Reyno del Pirú, etc., del Balthasar Ramírez (capellán en México, año de 1597). En el tomo II de la obra de Mujía se relata en p. 87 del comercio con cabezas trofeas entre los chiguanos.

NoRdensKiold, ERLAND. 1912-Indianerleben. Leipzig.

Pp. 137-141, comercio. Se trata de las tribus del Gran Chaco.

1919.1931-Comparative Ethnographical Studies,. tomos I-IX. Goteborg.

Varios de estos tomos tienen valiosa información del comercio, especialmente el primer tomo llamado An ethno-geographical analysis of the material culture of two Indian tribes in the Gran Chaco.

Relaciones Geográficas de Indias, tomos I-III. 1881-1897.

Esta obra clásica es una verdadera mina de informaciones del Perú en el siglo XVI.

Samanos, Juan de. 1844-Relación de los primeros descubrimientos de Francisco Pizarro y Diego de Almagro, sacada del códice número CXX de la Biblioteca Imperial de Viena. (Col. de Documentos Inéditos para la Historia de España, tomo V). Madrid.

En la p. 196 y sig. relata del encuentro que tenía el piloto Bartolomeo Ruiz con una balsa del Perú fuera de la costa. Este navío, que tomaba Ruiz, llevaba una carga de mercancías. Comp. R. C. Murphy: The Earliest Spanish advances southward, etc., en The Hispanic American Historical Review, tomo 21:2, pp. 2-28, Durham, N. Car. 1941. 
Schmidt, Max. 1921-Grundis der ethnologischen Volkwirtschaftslehre. T. 2: Der soziale Wirtschaftsprozess der Menschheit. Stuttgart.

Steinen, Karl voN DEN. 1894 -Unter den Naturvolkern Zentral-Brasiliens. Reiseschilderung und Ergebnisse der zweiten Schingú-Expedition 1887-1888. Berlín.

Pp. 333-334 del comercio, derecho, etc. P. 502 del valor que tenían flechas entre los bororó.

Whiffen, Thomas. 1915-The North-West Amazons. Notes of some months spent among Cannibal Tribes. London.

En p. 91 relata como son ciertas tribus especialistas en la manufactura de ciertas cosas. 